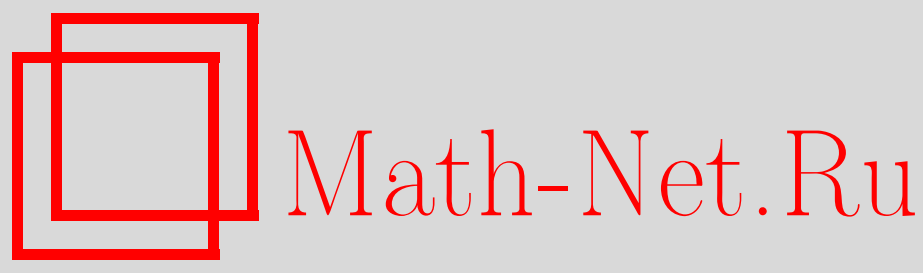

С. В. Талалов, Об описании кос в терминах спектральных задач первого порядка, ТМФ, 2009, том 159, номер 1, 58-63

DOI: https://doi.org/10.4213/tmf6332

Использование Общероссийского математического портала Math-Net.Ru подразумевает, что вы прочитали и согласны с пользовательским соглашением http://www.mathnet.ru/rus/agreement

Параметры загрузки:

IP : 54.205 .225 .156

26 апреля 2023 г., 13:53:47

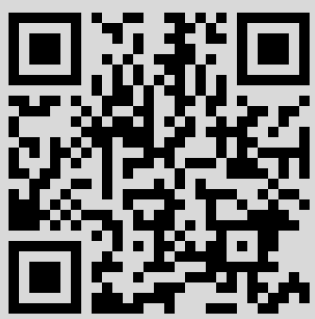




\title{
ОБ ОПИСАНИИ КОС В ТЕРМИНАХ СПЕКТРАЛЬНЫХ ЗАДАЧ ПЕРВОГО ПОРЯДКА
}

\begin{abstract}
Рассматривается представление крашеных кос мировыми линиями точек возврата бесконечной струны в пространстве $E_{1,2}$. Предложено описание кос парой спектральных задач первого порядка для матриц размера $2 \times 2$.
\end{abstract}

Ключевые слова: косы, трехмерные струны.

Различному описанию кос (см., например, [1]) в последнее время уделяется повышенное внимание, в том числе благодаря появлению этих объектов в моделях топологических квантовых компьютеров [2]. Настоящая статья является следствием обобщения и, в определенной степени, визуализации некоторых результатов, полученных ранее автором [3], [4]. Напомним кратко основные положения подхода к описанию релятивистской четырехмерной струны, развитому в работе [3]. Для того чтобы получить интересующие нас здесь движения струны в произвольной фиксированной плоскости (трехмерный случай), мы выполним соответствующую редукцию. Пусть $\mathcal{X}$ - множество локально-минимальных (т.е. имеющих нулевую среднюю кривизну) поверхностей в псевдоевклидовом пространстве $E_{1,3}$. Предполагается, что исследуемые поверхности, задаваемые радиус-вектором $X_{\mu}\left(\xi^{0}, \xi^{1}\right)$, не имеют границы, являются времениподобными почти всюду, а их параметризация $\left(\xi^{0}, \xi^{1}\right)$ является конформно-плоской:

$$
\partial_{+} \partial_{-} X_{\mu}=0, \quad\left(\partial_{ \pm} X_{\mu}\right)^{2}=0, \quad \xi_{ \pm}=\xi^{1} \pm \xi^{0}
$$

Такие объекты, как известно, возникают в теории релятивистских [5], а также космических [6] струн, поэтому термин "струна" мы будем использовать для бесконечной пространственноподобной кривой $X_{\mu}=X_{\mu}\left(\xi^{1}\right)$, мировой лист которой имеет указанные свойства. Что касается условий, налагаемых на поведение кривой при $\xi^{1} \rightarrow \pm \infty$, то мы сформулируем их позже; пока нам важна лишь локальная структура мирового листа. Имея в своем распоряжении пару (касательных) светоподобных векторов $\mathbf{e}_{ \pm}\left(\xi_{ \pm}\right)= \pm \partial_{ \pm} X$, всегда можно построить пару ортонормированных

*Тольяттинский государственный университет, Тольятти, Россия.

E-mail: svtalalov@tltsu.ru 
базисов $\mathbf{e}_{\nu \pm}\left(\xi_{ \pm}\right)$так, что выполняются равенства $\mathbf{e}_{ \pm}=\left(\mathbf{e}_{0 \pm} \mp \mathbf{e}_{3 \pm}\right) / 2$. Произвол, который здесь, конечно, возникает, будет устранен ниже. Важным объектом в предлагаемом подходе является $S L(2, C)$-значное поле $K\left(\xi^{0}, \xi^{1}\right)$, которое определено равенством

$$
\widehat{\mathbf{E}}_{+}=K \widehat{\mathbf{E}}_{-} K^{+}, \quad \widehat{\mathbf{E}}_{ \pm}=\mathbf{e}_{0_{ \pm}} \mathbf{1}_{2}-\sum_{i=1}^{3} \mathbf{e}_{i_{ \pm}} \boldsymbol{\sigma}_{i}
$$

Условие $K \in S L(2, \mathbb{R})$ отвечает редукции теории на подпространство $E_{1,2} \perp \mathbf{b}_{2}$, где $\mathbf{b}_{2}$ - постоянный пространственноподобный единичный вектор, так что $\mathbf{b}_{2}=$ $\mathbf{e}_{2+}=\mathbf{e}_{2-}$. Далее везде мы рассматриваем именно такую редукцию четырехмерного случая. Поскольку справедливы соотношения $\partial_{\mp} \hat{\mathbf{E}}_{ \pm}=0$, поле $K\left(\xi^{0}, \xi^{1}\right)$ удовлетворяет уравнению

$$
\partial_{+}\left(K^{-1} \partial_{-} K\right)=0
$$

которое, очевидно, есть частный случай уравнения Весса-Зумино-Новикова-Виттена. Далее, записывая для матрицы $K\left(\xi^{0}, \xi^{1}\right)$ разложение Гаусса

$$
K=\left(\begin{array}{cc}
1 & 0 \\
-\alpha_{+} & 1
\end{array}\right)\left(\begin{array}{cc}
e^{-\varphi / 2} & 0 \\
0 & e^{\varphi / 2}
\end{array}\right)\left(\begin{array}{cc}
1 & \alpha_{-} \\
0 & 1
\end{array}\right),
$$

однозначно определяем вещественные функции $\varphi\left(\xi^{0}, \xi^{1}\right)$ и $\alpha_{ \pm}\left(\xi^{0}, \xi^{1}\right)$. Данные функции являются, вообще говоря, сингулярными: разложение Гаусса не определено там, где главный минор $K_{11}=e^{-\varphi / 2}$ матрицы $K$ обращается в нуль. Именно эти сингулярности функции $\varphi$ соответствуют мировым линиям точек возврата струны, топология которых обсуждается в настоящей работе. Уравнение (2) совместно с разложением (3) приводит к системе второго порядка для полей $\varphi$ и $\alpha_{ \pm}$. Определим функции $\rho_{ \pm}=\left(\partial_{ \pm} \alpha_{\mp}\right) \mathrm{e}^{-\varphi}$. Они позволяют заменить в задаче Коши для указанной системы второго порядка набор начальных (роль “времени" играет параметр $\xi^{0}$ ) данных $\left(\varphi, \dot{\varphi}, \alpha_{ \pm}, \dot{\alpha}_{ \pm}\right)$на набор начальных данных $\left(\varphi, \dot{\varphi}, \alpha_{ \pm}, \rho_{ \pm}\right)$и рассматривать далее функции $\rho_{ \pm}$как динамические переменные. В итоге получаем следующую систему дифференциальных уравнений ${ }^{1)}$ :

$$
\begin{aligned}
\partial_{+} \partial_{-} \phi & =2 \rho_{+} \rho_{-} e^{\phi}, \\
\partial_{ \pm} \rho_{\mp} & =0, \\
\partial_{ \pm} \alpha_{\mp} & =\rho_{ \pm} e^{\phi} .
\end{aligned}
$$

Эта система имеет широкую группу инвариантности $\mathcal{G}$, которая включает в себя конформные преобразования

$$
\xi_{ \pm} \rightarrow \widetilde{\xi}_{ \pm}=A_{ \pm}\left(\xi_{ \pm}\right), \quad A^{\prime} \neq 0
$$

и обобщает соответствующую группу уравнения Лиувилля. В частности, в $\mathcal{G}$ входят преобразования полей $\varphi, \alpha_{ \pm}, \rho_{ \pm}$, отвечающие произволу в выборе базисов $\mathbf{e}_{\nu \pm}$. Подробности могут быть найдены в цитированных выше работах автора. Введение функций $\varphi$ и $\rho_{ \pm}$представляется естественным с дифференциально-геометрической

\footnotetext{
1) Данные уравнения с ограничениями $\rho_{ \pm} \geqslant 0$ ранее были использованы для построения системы взаимодействующих скалярного и спинорного полей в двумерном пространстве-времени [7].
} 
точки зрения. Это следует из явных выражений для первой I и второй II квадратичных форм мирового листа:

$$
\mathbf{I}=-\frac{1}{2} \mathrm{e}^{-\varphi} d \xi_{+} d \xi_{-}, \quad \mathbf{I I}=\rho_{+} d \xi_{+}^{2}-\rho_{-} d \xi_{-}^{2} .
$$

Нетривиальное, как показано в работах [3], [4], обобщение известного геометрического подхода в теории релятивистских струн [5] здесь заключается в том, что вторая квадратичная форма II допускает обращение коэффициентов $\rho_{ \pm}\left(\xi_{ \pm}\right)$в нуль как в отдельных точках, так и на интервалах. В этом случае, очевидно, преобразованиями (5) невозможно избавиться от данных функций в уравнении (4а) и преобразовать его к уравнению Лиувилля.

Далее мы выполняем факторизацию множества $\mathcal{X}$ по группе преобразований $\mathfrak{G}=$ $\mathcal{P} \times \mathcal{D} \times \mathcal{G}_{0}$, где $\mathcal{G}_{0} \subset \mathcal{G}($ см. [3]), а группы $\mathcal{P}$ и $\mathcal{D}$ - группы движений и масштабных преобразований пространства $E_{1,2}$ соответственно. Заметим, что преобразование (5) остается произвольным и нигде не фиксируется. Установлено, что соответствующее фактор-множество описывается парой спектральных задач первого порядка

$$
T_{ \pm}^{\prime}(\xi ; \lambda)+Q_{ \pm}(\xi) T_{ \pm}(\xi ; \lambda)=i \lambda \sigma_{3} T_{ \pm}(\xi ; \lambda)
$$

где $Q_{-}\left(\xi^{0}, \xi^{1}\right)=K^{-1} \partial_{-} K, Q_{+}\left(\xi^{0}, \xi^{1}\right)=-\left(\partial_{+} K\right) K^{-1}$. Матрица $K\left(\xi^{0}, \xi^{1}\right)$ представима через матрицы-решения $T_{ \pm}(\xi) \equiv T_{ \pm}(\xi ; 0)$ следующим образом: $K\left(\xi^{0}, \xi^{1}\right)=$ $T_{+}\left(\xi_{+}\right) T_{-}^{-1}\left(\xi_{-}\right)$. Преобразованиями из группы $\mathcal{G}_{0}$ матрицы $Q_{ \pm}-$коэффициенты спектральной задачи - можно привести к виду $Q_{ \pm}=-\rho_{ \pm} \boldsymbol{\sigma}_{ \pm}+\rho_{ \pm} \boldsymbol{\sigma}_{\mp}$; в дальнейшем в смежных классах $\mathcal{X} \backslash \mathfrak{G}$ выбираются только представители с такими матрицами $Q_{ \pm}$. Для применения аппарата обратной задачи рассеяния необходимо потребовать, чтобы функции $\rho_{ \pm}$принадлежали пространству Шварца. Это требование диктует асимптотические условия, о которых говорилось выше: мы рассматриваем мировые листы, плоские на пространственноподобных бесконечностях.

Касательные векторы $\mathbf{e}_{ \pm}$определяются явно по элементам $t_{i j \pm}$ матриц-решений $T_{ \pm}(\xi ; 0) \in S O(2):$

$$
\mathbf{e}_{ \pm}=\frac{1}{2} \mathbf{b}_{0}-\left(t_{i 1 \pm} t_{i 2 \pm}\right) \mathbf{b}_{1}-\frac{1}{2}\left(t_{i 1 \pm}^{2}-t_{i 2 \pm}^{2}\right) \mathbf{b}_{3}
$$

где знак \pm согласован с индексом $i$ согласно правилу $i=(3 \mp 1) / 2$, а тетрада $\mathbf{b}_{\mu}-$ некоторый постоянный ортонормированный базис в $E_{1,3}$ (напомним, мы рассматриваем редукцию $E_{1,2} \perp \mathbf{b}_{2}$ ). Таким образом, мы явно восстанавливаем радиус-вектор мирового листа (соответствующего представителя класса смежности) по решениям спектральных задач:

$$
\mathbf{X}\left(\xi^{0}, \xi^{1}\right)=\int_{0}^{\xi_{+}} \mathbf{e}_{+}(\xi) d \xi-\int_{0}^{\xi_{-}} \mathbf{e}_{-}(\xi) d \xi
$$

Давно известно [8], что струна в трехмерном пространстве-времени может иметь особенности типа точек возврата, которые не разрушаются в динамике. Один из результатов работы [3] состоит в том, что такие особенности соответствуют точкам дискретного спектра данных рассеяния линейных систем (6). В простейшем варианте, когда непрерывный спектр у спектральных задач (6) отсутствует (" $N$-солитонные" решения), а числа $N_{ \pm}$собственных значений равны единице, мировые линии 


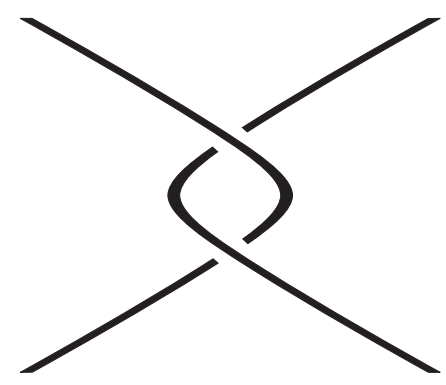

Рис. 1. Мировые линии точек возврата для $N_{ \pm}=1$.

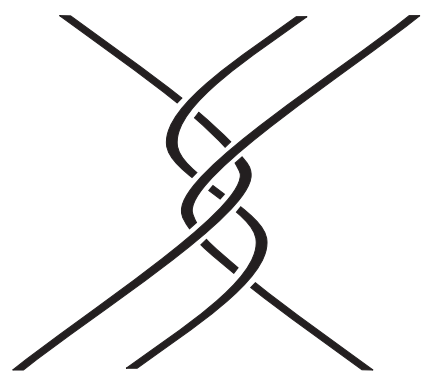

Рис. 2. Мировые линии для "трехсолитонного" случая (А).

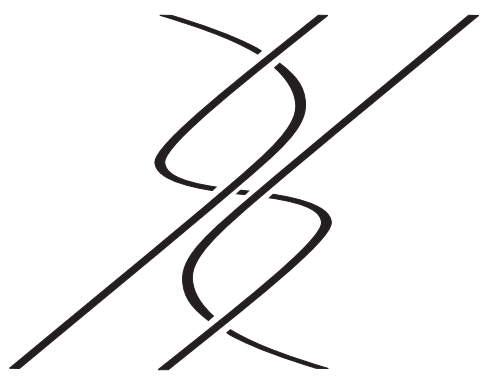

Рис. 3. Мировые линии для "трехсолитонного" случая (В).

точек возврата струны изображены на рис. 1. Для наглядности здесь отсутствует графическое изображение мирового листа (приведенное на рисунке для данного случая в статье [3]). Нетривиальность обсуждаемой конструкции видна уже при $N_{+}=2$ и $N_{-}=1$. Соответствующие варианты кос мировых линий приведены на рис. 2 и рис. 3. Видно, что данные косы топологически неэквивалентны. Сделаем по этому поводу некоторые пояснения. В приведенных примерах для коэффициентов $\rho_{ \pm}$выполняется строгое неравенство $\rho_{+} \rho_{-}>0$, так что (в данном частном случае) мировые линии точек возврата могут быть сопоставлены сингулярным решениям уравнения Лиувилля для функции $\varphi\left(\xi^{0}, \xi^{1}\right)$, определяющей метрику мирового листа. Сингулярности уравнения Лиувилля в плоскости $\left(\xi^{0}, \xi^{1}\right)$ не пересекаются [9]. Отсюда, однако, не следует невозможность пересечения рассматриваемых мировых линий: сама струна, как вложение прямой $\xi^{0}=$ const пространства $E_{1,1}$ в двумерную плоскость $X^{0}=$ const пространства $E_{1,2}$, может иметь пересечения.

Рассмотрим отличие кос на рис. 2 и рис. 3 с точки зрения спектральных задач (6). Каждая точка дискретного спектра каждого из уравнений (6) характеризуется, как известно [10], парой констант $\left(a_{n \pm}, c_{n \pm}\right)$, где $a_{n \pm}-$ собственное значение, а $c_{n \pm}-$ коэффициент, связывающий определенные столбцы матрицы решений $T_{ \pm}(\xi ; \lambda)$ при $\lambda=a_{n \pm}$. Вещественность функций $\rho_{ \pm}\left(\xi_{ \pm}\right)$означает, что точки спектра $a_{n}$ (для каждого знака из \pm ) либо мнимые, либо образуют симметричные относительно мнимой оси пары [11]. Точкам возврата струны соответствуют мнимые величины $a_{n}$, поэтому далее всегда $a_{n}=i \varkappa_{n}$, где вещественная константа $\varkappa_{n}>0$. На рис. 2 и 3 изображены мировые линии точек возврата, построенные в соответ- 
ствии с одним из двух предположений:

$$
\text { (A): } \varkappa_{1-}<\varkappa_{1+}<\varkappa_{2+}, \quad(\mathrm{B}): \varkappa_{1+}<\varkappa_{2+}<\varkappa_{1-} .
$$

В обоих случаях $c_{1-}<0, c_{1+}<0, c_{2+}>0$. Приведенные примеры показывают, что различные соотношения между константами дискретного спектра приводят, вообще говоря, к топологически разным косам. При деформации спектра в какой-то момент в косе возникает сингулярный перекресток (точка пересечения нитей), что соответствует некоторому соотношению $\Phi(a, c)=0$ в пространстве параметров $a_{i \pm}, c_{i \pm}$. Интересно заметить также, что если в каждом из случаев (A) и (B) у констант $c_{1+}$ и $c_{2+}$ поменять знаки, то мы получим струнные конфигурации с $\rho_{+} \rho_{-}<0$, которые могут иметь точки возврата, исчезающие и возникающие (парами) в динамике. Соответствующие мировые линии в данном случае косу не образуют.

В последнее время высказываются новые идеи об элементарных частицах как о дефектах струнного конденсата [12]. При этом, например, отмечается, что концы открытых струн могут быть интерпретированы как фермионы. По-видимому, такая же интерпретация допустима и для точек возврата струн ${ }^{2)}$ (в том числе бесконечных и замкнутых). В этой связи естественно предположить, что пересечения рассматриваемых мировых линий при правильном выборе квантования станут невозможными. Это будет означать, что в квантовой теории таких "квазичастиц на плоскости" существуют топологически неэквивалентные классы.

Благодарности. Автор благодарен В. С. Лоозе за существенную помощь в компьютерной визуализации представленных здесь объектов.

\section{Список литературы}

[1] В. О. Мантуров, Теория узлов, РХД, Москва-Ижевск, 2005.

[2] J. Preskill, Lecture Notes for Physics 219: Quantum Computation, http://www.theory. caltech.edu/ preskill/ph219/topological.pdf.

[3] С. В. Талалов, ТМФ, 152:3 (2007), 430-439.

[4] С. В. Талалов, ТМФ, 123:1 (2000), 38-43; J. Phys. A, 22 (1989), 2275.

[5] Б. М. Барбашов, В. В. Нестеренко, Модель релятивистской струны в физике адронов, Энергоатомиздат, М., 1987.

[6] M. R. Anderson, The Mathematical Theory of Cosmic Strings. Cosmic Strings in the Wire Approximation, Ser. High Energy Phys. Cosmol. Gravit., IOP, Bristol, 2003.

[7] А. К. Погребков, С. В. Талалов, ТМФ, 70:3 (1987), 342-247.

[8] Г. П. Пронько, А. В. Разумов, Л. Д. Соловьев, ЭЧАЯ, 14:3 (1983), 558-577.

[9] Г. П. Джорджадзе, А. К. Погребков, М. К. Поливанов, ТМФ, 40:2 (1979), 221-234.

[10] Л. А. Тахтаджян, Л. Д. Фаддеев, Гамильтонов подход в теории солитонов, Наука, М., 1986.

[11] С. В. Талалов, ТМФ, 71:3 (1987), 357-369.

[12] M. Levin, X.-G. Wen, Phys. Rev. Lett., 96:11 (2006), 110405.

[13] A. K. Pogrebkov, M. K. Polivanov, "The Liouville and Sinh-Gordon Equations. Singular Solutions, Dynamics of Singularities and the Inverse Problem Method", Mathematical Physics Reviews, Sov. Sci. Rev. Sect. C, 5, ed. S. P. Novikov, Harwood Academic, Chur, 1985, 197-272.

Поступила в редакцию 26.06.2008, после доработки 11.08.2008

\footnotetext{
2) Тот факт, что сингулярности решений уравнения Лиувилля демонстрируют "фермионные” свойства уже на классическом уровне, известен давно [13].
} 